\title{
Role of sarcoplasmic reticulum junctional proteins in skeletal muscle strength
}

\author{
Barbara Mosca ${ }^{1}$, Osvaldo Delbono ${ }^{2}$, Maria Laura Messi ${ }^{2}$, Leda Bergamelli', Mirko Vukcevic ${ }^{3}$, Ruben Lopez ${ }^{1}$, \\ Susan Treves ${ }^{1,3}$, Miyuki Nishi ${ }^{4}$, Hiroshi Takeshima ${ }^{4}$, Francesco Zorzato ${ }^{1,3^{*}}$ \\ From 33rd Annual Meeting of the European Malignant Hyperthermia Group (EMHG) \\ Würzburg, Germany. 15-17 May 2014
}

\section{Background}

Skeletal muscle constitutes approximately $40 \%$ of body mass, and age-induced decrease of muscle strength impinge on daily activities and on normal social life in the elderly. Loss of muscle strength has been recognised as a debilitating and life threatening condition also in cachexia in cancer patients and in clinical conditions associated with prolonged bed rest. Skeletal muscle dihydropyridine receptors (Cav1.1) act as $\mathrm{Ca} 2+$ channels and voltage sensors to initiate muscle contraction by activating ryanodine receptors, the $\mathrm{Ca} 2+$ release channels of the sarcoplasmic reticulum. Cav1.1 activity is enhanced by a retrograde stimulatory signal delivered by the ryanodine receptor. JP45 is a membrane protein interacting with Cav1.1 and the sarcoplasmic reticulum $\mathrm{Ca} 2+$ storage protein calsequestrin (CASQ1).

We hypothesized that JP45 and CASQ1 form a signalling pathway which modulates Cav1.1 channel activity.

\section{Materials and methods}

We isolated flexor digitorum brevis (FDB) muscle fibres from JP45 and CASQ1 double knock-out mice (DKO) and tested whether there were differences in $\mathrm{Ca} 2+$ homeostasis between the different mouse lines.

\section{Results}

Our results show that $\mathrm{Ca} 2+$ transients evoked by tetanic stimulation in DKO fibres, result from massive $\mathrm{Ca} 2+$ influx due to enhanced Cav1.1 channel activity. This enhanced activity causes an increase of muscle strength both in vitro and in vivo.

\section{Conclusions}

We conclude that skeletal muscle contraction is strengthened through the modulation of Cav1.1 channel activity by JP45 and CASQ1.

\section{Acknowledgements}

This work was supported by funds from Swiss Muscle foundation, A.F.M., S.N. $\mathrm{F}$ and Department of Biomedicine University Hospital Basel. This study was also supported by Research Grant no. GGP08153 from the Italian Telethon ONLUS Foundation to F.P. and grants from the NIH/NIA (AG13934 and AG15820) to O.D.

\section{Authors' details}

'Department of Life Sciences and Biotechnology, General Pathology section, University of Ferrara, Ferrara, 44100, Italy. ${ }^{2}$ Department of Physiology and Pharmacology, Wake Forest University School of Medicine, Winston-Salem, N. C. 27157, USA. ${ }^{3}$ Departments of Biomedicine and Anesthesiology, Basel University Hospital, Basel, 4031, Switzerland. ${ }^{4}$ Department of Biological Chemistry, Graduate School of Pharmacological Sciences, Kyoto University, Tokyo, 113-0033, Japan.

Published: 18 August 2014

\section{doi:10.1186/1471-2253-14-S1-A19}

Cite this article as: Mosca et al:: Role of sarcoplasmic reticulum junctional proteins in skeletal muscle strength. BMC Anesthesiology 2014 14(Suppl 1):A19. 